\title{
Silencing of ATPase Inhibitory Factor 1 Inhibits Cell Growth via Cell Cycle Arrest in Bladder Cancer
}

\author{
Shihu Wei ${ }^{a}$ Hideo Fukuhara ${ }^{a}$ Chiaki Kawada $^{a}$ Atsushi Kurabayashi ${ }^{b}$ \\ Mutsuo Furihata $^{b}$ Shun-ichiro Ogura ${ }^{c}$ Keiji Inoue $^{a}$ Taro Shuin $^{b}$ \\ Departments of a Urology and b Pathology, Kochi Medical School, Nankoku, Kochi, and ' $G$ raduate School of \\ Bioscience and Biotechnology, Tokyo Institute of Technology, Yokohama, Japan
}

\section{Key Words}

ATPase inhibitory factor $1 \cdot$ Bladder cancer $\cdot$ Cell cycle arrest $\cdot$ Knockdown $\cdot$ Proliferation

\begin{abstract}
Objective: The role of the ATPase inhibitory factor 1 (IF1) is inhibit the hydrolase activity of $F_{1} F_{0}$-ATPase when oxidative phosphorylation is impaired. It has been demonstrated that IF1 is overexpressed in various carcinomas and mediates tumor cell activities, but the detailed mechanisms of IF1-mediated tumor progression and the link between IF1 and cell cycle progression remain unclear. Herein, we aimed to investigate the potential role of IF1 in cell cycle progression of human bladder cancer (BCa). Methods: The expression of IF1 was analyzed by immunohistochemistry in tumor tissues. Western blot was used to detect protein expression in the cells. Cell proliferation was determined by MTT and colony formation assays. The cell cycle was analyzed using flow cytometry. Results: We firstly showed IF1 was overexpressed in $\mathrm{BCa}$. Silencing of IF1 by small interfering RNA led to a significant decrease in cell proliferation and migration in T24 and UM-UC-3 cells. Importantly, IF1 knockdown caused cell
\end{abstract}

cycle arrest at $G_{0} / G_{1}$ stage and decreased the protein level of cyclin E/cyclin-dependent kinases (cdk) 2 and/or cyclin D/cdk4/cdk6. Conclusion: These results suggest the inhibitory effect of IF1 knockdown on BCa cell proliferation is via the suppression of cyclins and cdks related to $G_{1} / S$ transition and then induction of $G_{0} / G_{1}$ arrest, and firstly indicate IF1 mediates the tumor cell cycle. We concluded that IF1 may be a novel therapeutic target for $\mathrm{BCa}$.

ㄷ) 2015 S. Karger AG, Basel

\section{Introduction}

The endogenous $\mathrm{F}_{1} \mathrm{~F}_{\mathrm{o}}$-ATPase inhibitory factor 1 (IF1) is a naturally occurring protein in mammals and predominantly located inside the mitochondrial matrix [1]. When mitochondrial respiratory activity is impaired or a cell is deprived of oxygen, IF1 becomes active as a dimer and then binds to the $\beta$-subunit of $\mathrm{F}_{1} \mathrm{~F}_{\mathrm{o}}-\mathrm{ATPase}\left(\beta \mathrm{F}_{1}\right)$, and finally acts to block the ATP-hydrolyzing activity of the $\mathrm{F}_{1} \mathrm{~F}_{\mathrm{o}}$-ATPase [2]. Although the physiological role of IF1 has been clarified several decades ago, it is now regarded as a pleiotropic factor exerting diverse cellular effects [3-

\section{KARGER 125\%}

(c) 2015 S. Karger AG, Base

$1015-2008 / 15 / 0825-0224 \$ 39.50 / 0$
Keiji Inoue, $\mathrm{MD}, \mathrm{PhD}$

Department of Urology

Kochi Medical School

Kohasu, Oko, Nankoku, Kochi, 783-8505 (Japan)

E-Mail keiji@kochi-u.ac.jp 
Table 1. The characteristics of the BCa patients

\begin{tabular}{lc}
\hline Mean age, years (range) & $68.7(41-83)$ \\
Gender & \\
Male & 13 \\
Female & 2 \\
Pathological stage, n (\%) & \\
pTis & $2(13.3)$ \\
pT1 & $4(26.7)$ \\
pT2 & $5(33.3)$ \\
pT3 & $4(26.7)$ \\
Grade, $\mathrm{n}(\%)$ & \\
Low grade & $0(0)$ \\
High grade & $15(100)$
\end{tabular}

5], and many of them are potentially related to tumorigenesis, including cell proliferation, migration, invasion and angiogenesis $[6,7]$.

Some studies show that IF1 is upregulated in various common human cancers, and increased expression of IF1 in hepatocellular carcinoma predicts poor survival and disease recurrence after surgery [7-9]. But other reports claim that a low tumor expression of IF1 predicts the worst prognosis overall for breast and colon cancer patients [10]. These results suggest that the functional effect of IF1 in human tumors is complicated. Thus, more mechanistic studies are required to elucidate the role of IF1 in human carcinomas.

Dysregulation of cellular proliferation is a hallmark of cancer [11]. Cell proliferation relies on the activation of cyclins, which in turn bind to cyclin-dependent kinases (cdks), forming complexes that drive progression through the different phases of the cell cycle and ultimately lead to cell growth [12]. This is coupled to regulatory mechanisms by cdk inhibitors that allow or prevent cells to progress through the cell cycle. Although previous studies suggest IF1 mediates tumor cell activities, such as cell proliferation, invasion and response to toxic agents $[6,7$, 13], it still remains unclear whether IF1 is linked to cell cycle progression. In this study, we investigated the potential role of IF 1 on cell cycle progression using human bladder cancer $(\mathrm{BCa})$ cells in order to reveal new mechanisms of IF1-mediated tumor progression.

\section{Materials and Methods}

Tissue Samples, Histology and Immunohistochemistry

We obtained $\mathrm{BCa}$ specimens $(\mathrm{n}=15)$ from patients undergoing transurethral resection or radical cystectomy at the Kochi Medical University Hospital (table 1). The current research was approved by the Ethical Committee of the Kochi Medical School (No. 26). Frozen tissues were cut into $10-\mu \mathrm{m}$-thick sections. Slides were then stained with hematoxylin and eosin to distinguish normal from tumor tissue. IF1 expression was detected by immunohistochemistry. The tissue sections were deparaffinized in Lemosol ${ }^{\circledR} \mathrm{A}$, rehydrated in graded ethanol series and transferred to phosphatebuffered saline (PBS). Endogenous peroxidases were blocked by incubation in $0.3 \%$ hydrogen peroxide in PBS for $30 \mathrm{~min}$ at room temperature. The samples were washed three times with PBS and then treated with citric acid buffer ( $\mathrm{pH}$ 6.0) under microwave heating. The samples were incubated overnight at $4^{\circ} \mathrm{C}$ with the appropriate dilution (1:100) of monoclonal IF1 antibody (Santa Cruz Biotechnology Inc.). They were then rinsed three times with PBS and incubated for 15 min with biotinylated link antibody and streptavidin-HRP (DakoCytomation Co. Ltd., Kyoto, Japan). The slides were rinsed with PBS and incubated for $5 \mathrm{~min}$ with diaminobenzidine. The sections were washed three times with PBS and counterstained with hematoxylin.

\section{Cell Lines, Culture Conditions and Transfection}

All human BCa cells except T24 cells were grown as monolayers in Dulbecco's modified Eagle's medium supplemented with $10 \%$ fetal bovine serum (FBS). T24 cells were cultured in F12 medium supplemented with $10 \%$ FBS. Human bladder epithelial cells from the apex region (HBEC-A) and dome region (HBEC-D) were purchased from Kurabo Industries Ltd. and maintained in UroLife ${ }^{\mathrm{TM}}$ A basal medium and LifeFactors, and UroLife ${ }^{\mathrm{TM}} \mathrm{D}$ complete medium (Lifeline Cell Technology, Frederick, Md., USA), respectively. These cells were incubated at $37^{\circ} \mathrm{C}$ in a $5 \% \mathrm{CO}_{2}$ environment. Small interfering RNA (siRNA; Qiagen S100908075) was used to suppress the expression of IF1, and transfection was carried out using HiPerFect transfection reagent according to the manufacturer's fast-forward protocol. One milliliter medium contains $5 \mu \mathrm{l} \mathrm{HiPer-}$ Fect transfection reagent and 5 pmol IF1 or control siRNA.

\section{Cell Proliferation and Colony-Forming Assay}

Cell proliferation was determined by 3-(4, 5-dimethyl-thiogol2-yl)-2, 5-diplenyltetrazolium (MTT) assay, as we previously reported [14]. Colony formation was assessed as described [15]. Briefly, cells transfected with IF1 siRNA (SiIF1) or control siRNA (SiCL) were plated in six-well plates at a density of 300 cells/well. Plates were incubated for 10 days, and colonies were fixed in $70 \%$ ethanol and stained with crystal violet.

\section{Cell Migration Assay}

Cell migration was determined using the wound healing assay, as described previously [16]. Briefly, cells transfected with SiIF1 or SiCL were plated in 24-well plates at a density of 5,000 cells/well. After incubation for $24 \mathrm{~h}$, wound injury was made with the tip of a sterile micropipette, and the detached cells were removed by washing with fresh medium. Cells were then incubated and allowed to migrate for another $24 \mathrm{~h}$. Photographs were taken and the migration index was calculated as follows: migration index $=[($ initial wound width - width of the wound at the time point tested)/ initial wound width] $\times 100 \%$.

Flow-Cytometric Analysis

After transfection, cells were trypsinized and washed with PBS. Cells were then stained with the bromodeoxyuridine (BrdU) flow kit (BD Biosciences) to determine the cell cycle according to the 


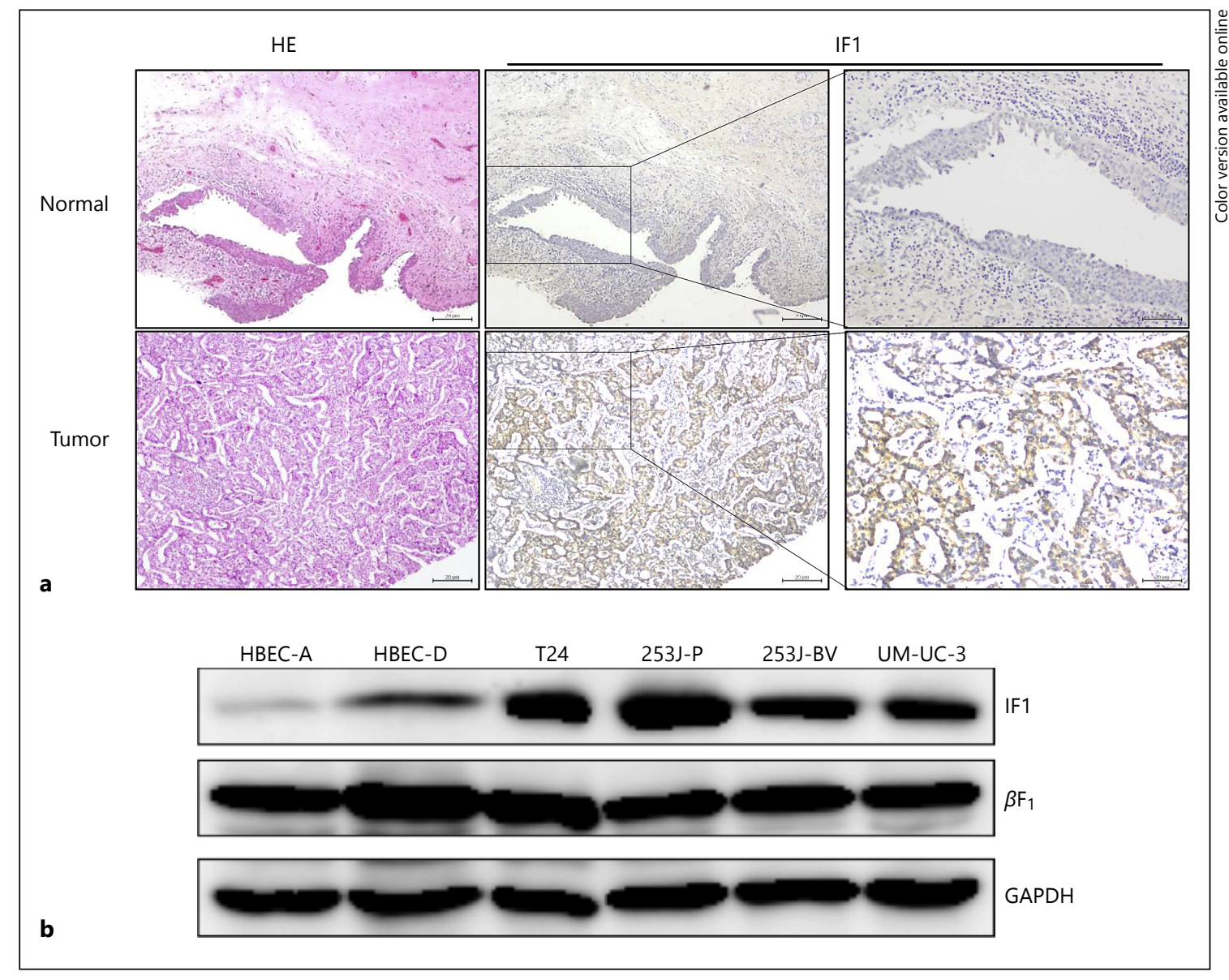

Fig. 1. IF1 is upregulated in BCa tissues and cell lines. a Representative IF1 expression levels are shown in normal bladder and BCa tissues by immunohistochemistry. Scale bars $=20 \mu \mathrm{m}$. b IF1 expression in human normal bladder and BCa cell lines determined by Western blots.

manufacturer's protocol. Annexin V and propidium iodide (PI) dyes were used to detect apoptosis. Cells were stained with $100 \mathrm{nM}$ tetramethylrhodamine methyl ester (TMRM) and $5 \mu \mathrm{M}$ MitoSOX for 15 min to determine mitochondrial membrane potential $\left(\Delta \Psi_{\mathrm{m}}\right)$ and reactive oxygen species (ROS), respectively. The stained cells were processed for LSRFortessa or FACScan (BD Biosciences). A minimum 10,000 cells per sample were collected and subsequently analyzed with FlowJo software.

\section{Western Blot}

Cells were harvested and then lysed by lysis buffer with protease inhibitor cocktail. Proteins from the cell lysate were performed on SDS-PAGE and transferred onto polyvinylidene difluoride membranes. After blocking, the membranes were probed with the appropriate antibodies against IF1, $\beta \mathrm{F}_{1}$ (Abcam plc., Cambridge, UK), cyclin D3 (DSC-22), cyclin E (HE12), cyclin-dependent kinase (cdk) 2 (D-12), cdk4 (DCS-156), cdk6 (DSC-83) and glyceraldehyde 3-phosphate dehydrogenase (GAPDH; Abcam), and then detected by enhanced chemiluminescence assay (Pierce Biotechnology, Rockford, Ill., USA). All other antibodies were purchased from Santa Cruz Biotechnology Inc.

\section{Statistics}

Data are expressed as means and SD. Student's independent two-sample $t$ test was used for comparisons. A value of $\mathrm{p}<0.05$ was considered statistically significant.

\section{Results}

\section{IF1 Expression Is Upregulated in Human $B C a$}

We firstly examined the expression of IF1 in $15 \mathrm{BCa}$ tissue samples and 12 corresponding adjacent noncancerous bladder tissues of them by immunostaining using IF1 antibody. Immunohistochemistry (fig. 1a) demonstrated that the expression of IF1 was negligible in normal bladder epithelial cells, but significantly increased in $\mathrm{BCa}$ tissue samples. The relative expression of IF1 in BCa cell lines indicated a very large variability by Western blot (data not shown), but was upregulated compared to the 


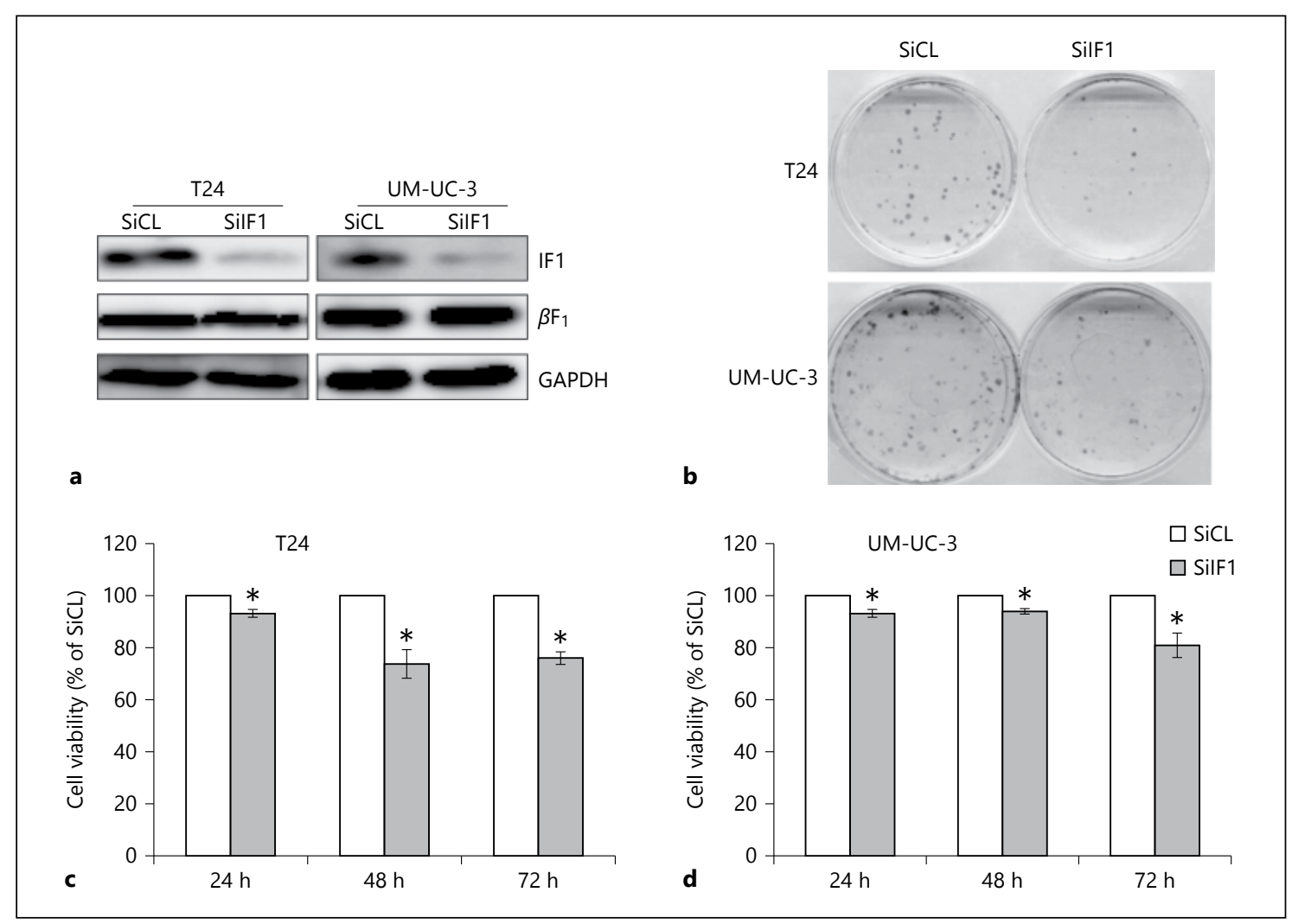

Fig. 2. Knockdown of IF1 induces BCa cell growth inhibition. a SiIF1 successfully knocked down IF1 expression in T24 and UMUC-3 cells. b Knockdown of IF1 led to a significant reduction in colony formation. c, $\mathbf{d}$ Knockdown of IF1 led to inhibition of pro-

normal bladder cell lines (fig. 1b). The resulting data showed overexpression of IF1 in BCa tissues and cells compared to the normal bladder tissues and cells.

\section{Knockdown of IF1 Expression Induces Cell Growth and Migration Inhibition in $\mathrm{BCa}$ Cells}

Given the increased expression of IF1 in BCa tissues and cell lines, we then determined whether IF1 inhibition affected BCa cell growth in vitro using T24 and UMUC-3 cells, which have relatively high proliferative and invasion abilities. Cells were transfected with SiCL or SiIF1, and SiIF1 transfection successfully reduced the expression of IF1 protein (fig. 2a). Knockdown of IF1 resulted in a decreased number of BCa colonies compared with SiCL transfection (fig. 2b). In addition, IF1 knockdown cells showed decreased cell viability. Cell viability was reduced by 7, 27 and $25 \%$ in T24 cells, and 14, 9 and $20 \%$ in UM-UC-3 cells after transfection for 24, 48 and $72 \mathrm{~h}$, respectively (fig. 2c, d). Taken together, these results indicated silencing of IF1 suppressed BCa cell growth.

ATPase Inhibitory Factor 1 in Bladder Cancer liferation of T24 (c) and UM-UC-3 cells (d) determined by MTT assay. ${ }^{*} \mathrm{p}<0.05$ vs. SiCL. Three independent experiments were performed in quadruplicate.

Elevated migration heightens the metastatic potential of cancer cells. This may be independent of cell-proliferative rates. Therefore, we investigated the effect of IF1 knockdown on $\mathrm{BCa}$ cell migration determined by a wound healing assay. As shown in figure $3 \mathrm{a}$, the knockdown of IF1 inhibited the migration of T24. A similar result was also found in UM-UC-3 cells (fig. 3b). The migration index of SiIF1 cells was $29 \%$ compared to $52 \%$ in SiCL cells in T24 cells. In UM-UC- 3 cells, the migration index of SiIF1 cells was reduced by $17 \%$ (from $49 \%$ to $32 \%$; fig. 3c). These data suggested that silencing of IF1 also suppressed the cell migration in BCa cells.

\section{Knockdown of IF1 Expression Induces $G_{0} / G_{1}$ Cell Cycle Arrest in $\mathrm{BCa}$ Cells}

To examine the mechanism by which IF1 inhibition may affect cell growth, cells were transfected with SiIF1 or SiCL, and cell cycle analysis was then carried out. After BrdU staining, flow-cytometric analysis revealed knockdown of IF1 significantly decreased the S-phase popula- 
tion from 28.9 to $8.6 \%$ and increased $\mathrm{G}_{0} / \mathrm{G}_{1}$ fraction from 54.3 to $72.6 \%$, indicating a $G_{0} / G_{1}$ cell cycle arrest in $T 24$ cells (fig. 4a, b). A similar result was also found in UMUC-3 cells. IF1 knockdown decreased the percentage of UM-UC-3 cells in S phase by nearly $21 \%$ and increased $\mathrm{G}_{0} / \mathrm{G}_{1}$ population from 48.9 to $72.0 \%$ (fig. $4 \mathrm{c}, \mathrm{d}$ ). In addition, there was no significant increase in apoptosis upon IF1 knockdown in T24 and UM-UC-3 cells detected by BrdU/7-AAD and annexin V/PI staining (data not shown). Taken together, these results suggested silencing of IF1 by siRNA was associated with a block in cell cycle progression and not increased apoptosis in BCa cells.

To elucidate the molecular changes associated with the observed $\mathrm{G}_{0} / \mathrm{G}_{1}$ cell cycle arrest, we determined the expression of a panel of key regulatory proteins of the cell cycle machinery by Western blot analysis (fig. 4e). Inhibition of IF1 in T24 and UM-UC-3 cells resulted in the suppression of $\mathrm{G}_{1} / \mathrm{S}$ transition molecules cyclin $\mathrm{E}, \mathrm{cdk} 2$, cdk 4 and cdk6. In addition, IF1 knockdown also decreased the protein level of cyclin D and increased the expression of cdk inhibitor p21 in UM-UC-3 cells. However, converse results that silencing of IF1 increased cyclin D expression and decreased p21 expression were found in T24 cells. Thus, our data suggested that the inhibition of IF1 prevents urothelial cancer cell cycle progression from $G_{1}$ to $S$ phase at least through the regulation of key cell cycle factors (fig. $4 \mathrm{f}$ ).

\section{Knockdown of IF1 Expression Increases Mitochondrial ROS in BCa Cells}

As mitochondria play a critical role in cell fate and IF 1 could regulate mitochondrial function [17], we next analyzed the effect of IF1 knockdown on mitochondrial $\Delta \Psi_{\mathrm{m}}$ and ROS. Consistent with the previous report by Formentini et al. [6], SiIF1-mediated silencing of IF1 exhibited a marginal effect on mitochondrial $\Delta \Psi_{\mathrm{m}}$ (fig. 5a, b). However, after MitoSOX staining, flow-cytometric analysis showed enhanced fluorescence intensity in SiIF1 cells compared to SiCL cells in T24 and UM-UC-3 cells (fig. 5c, d), indicating IF1 knockdown led to an increase in mitochondrial ROS of BCa cells.

\section{Discussion}

IF1 is a highly conserved protein encoded in the nuclear ATPIF1 gene. It has unique capacity to inhibit the hydrolase activity of $F_{1} F_{0}$-ATP synthase, which is its reverse role. IF1 is known to play a major role in heme biosynthesis and mitochondrial respiratory chain dysfunction [3, 18], and, moreover, has been linked to different cancer

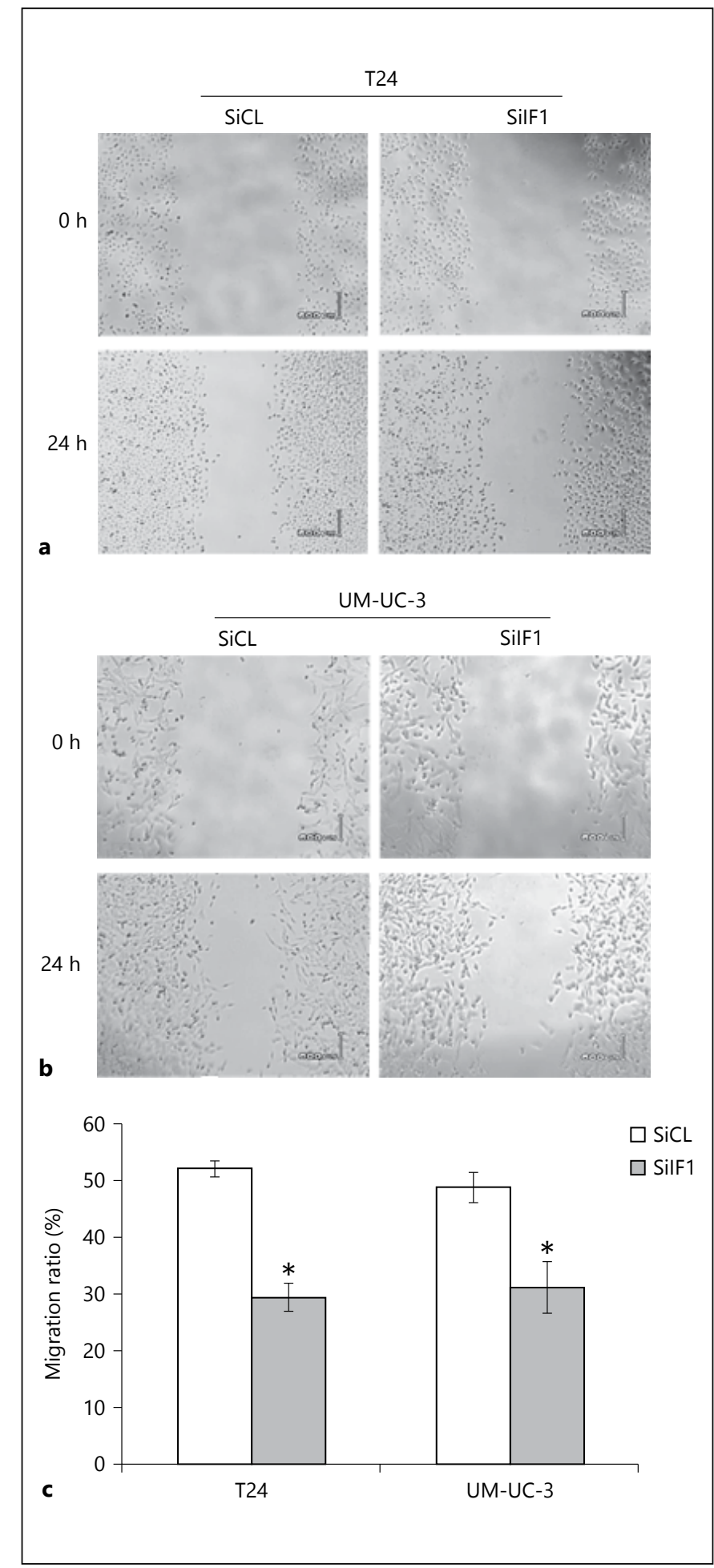

Fig. 3. Knockdown of IF1 inhibits BCa cell migration. a, b Representative images in T24 (a) and UM-UC-3 cells (b) determined by wound healing assay. Scale bars $=600 \mu \mathrm{m}$. c Knockdown of IF1 led to a slower migration of T24 and UM-UC-3 cells $24 \mathrm{~h}$ after making the scratches. ${ }^{*} \mathrm{p}<0.05$ vs. SiCL. The experiments were repeated three times. 


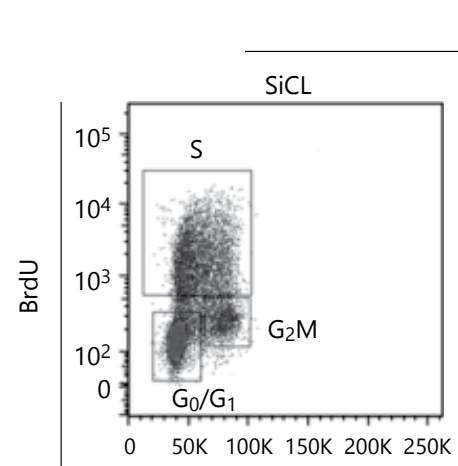

T24

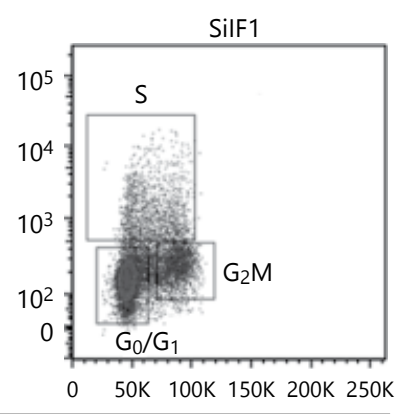

a

7-AAD

UM-UC-3

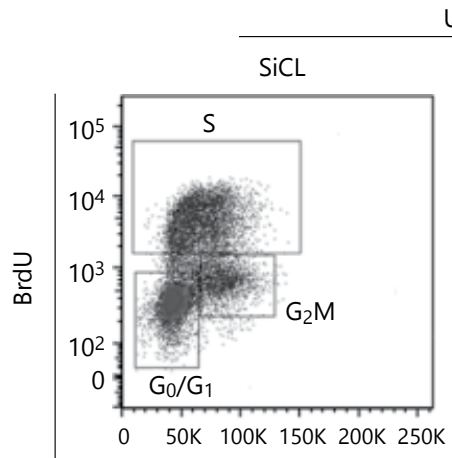

c
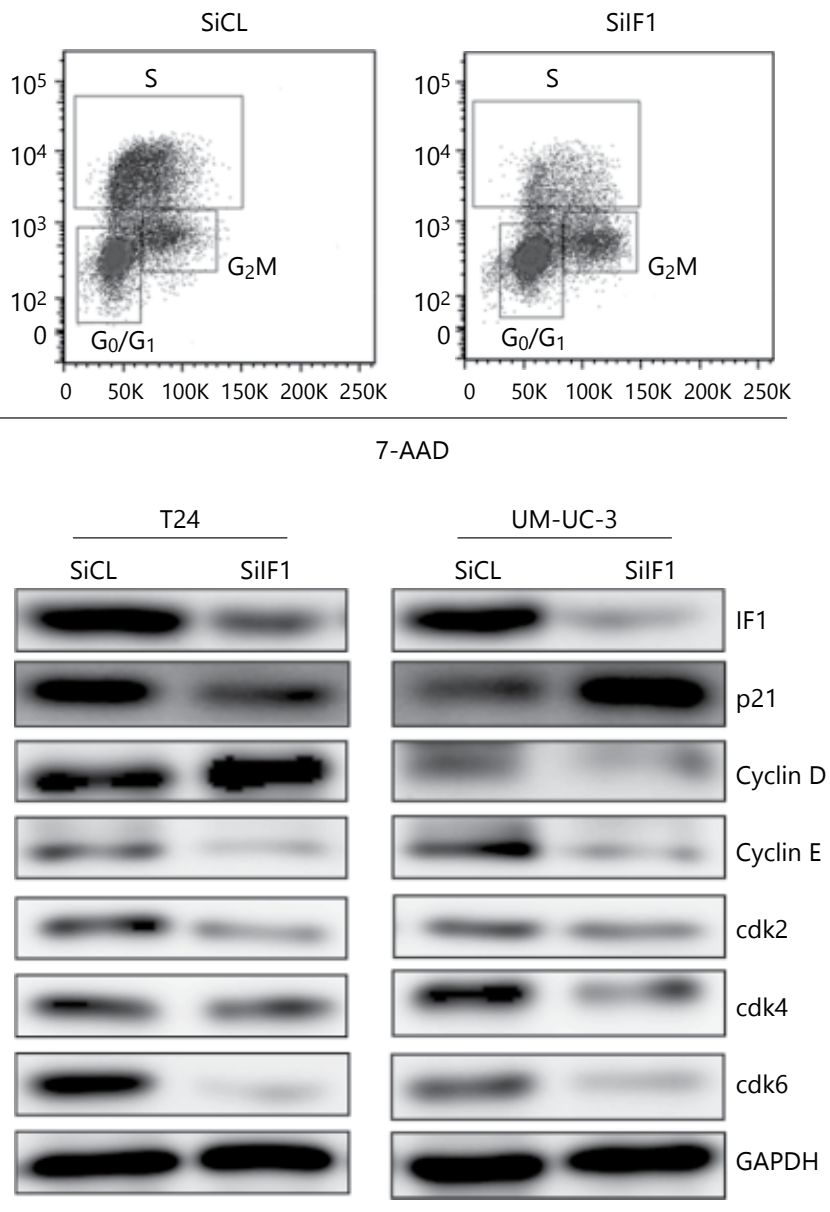

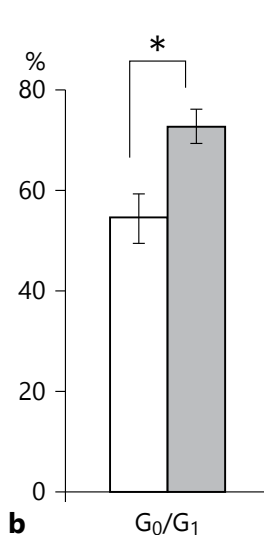

$\mathrm{T} 24$

b
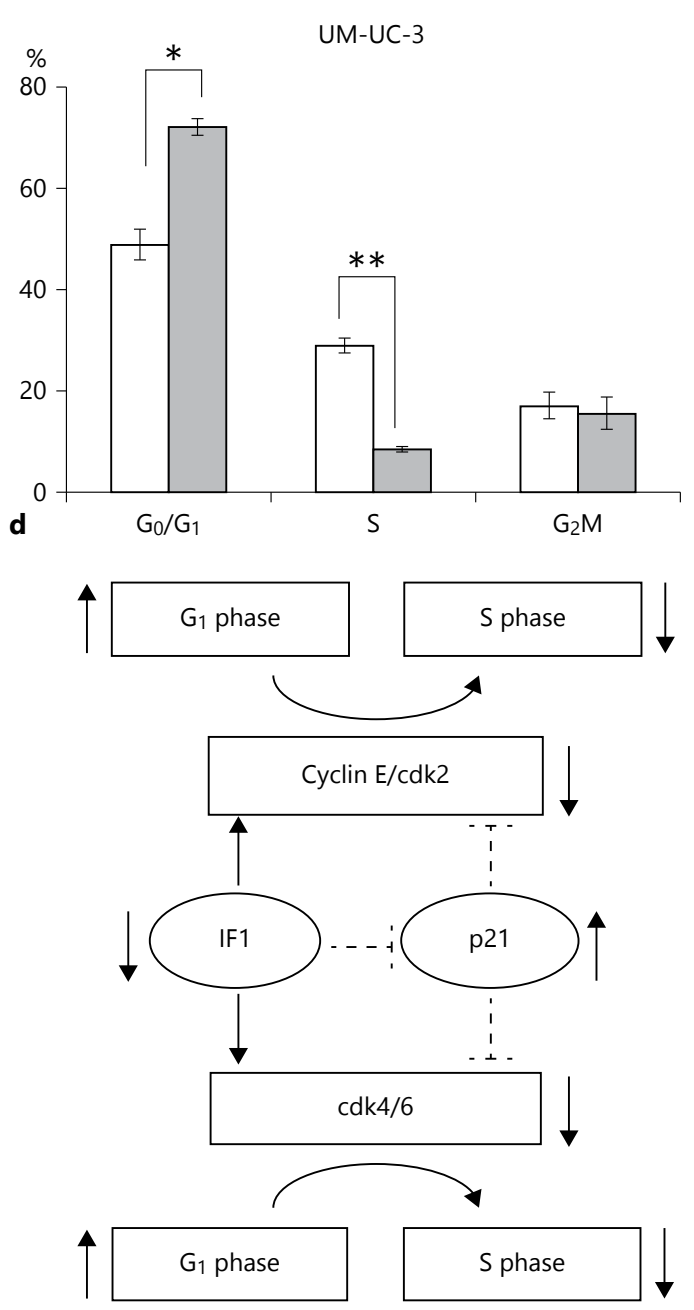

f

$\square \mathrm{SiCL}$

$\square$ SilF1

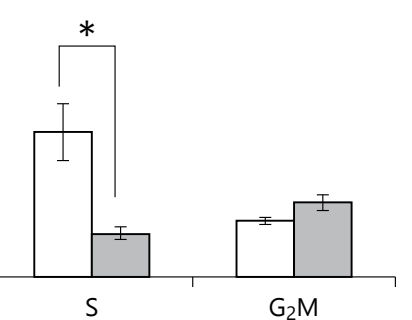

UM-UC-3

e

Fig. 4. Knockdown of IF1 induces BCa cell cycle arrest. a, c Representative dot plots in T24 (a) and UM-UC-3 cells (c) determined by flow cytometry after staining with BrdU and 7-AAD. b, d Knockdown of IF1 induced a $\mathrm{G}_{0} / \mathrm{G}_{1}$-phase arrest in T24 (b) and UM-UC-3 cells (d). ${ }^{*} \mathrm{p}<0.05,{ }^{* *} \mathrm{p}<0.01$, vs. SiCL. The experiments were repeated three times. e Expression levels of sev- eral known $G_{1} / S$ transition regulatory factors were analyzed by Western blot. $\mathbf{f}$ Schematic representation of the molecular mechanism proposed in the regulation of $\mathrm{G}_{0} / \mathrm{G}_{1}$-phase arrest via the downregulation of the cyclin/cdk complex with (dot lines) or without the p21-mediated pathway after IF1 inhibition in BCa cells. 


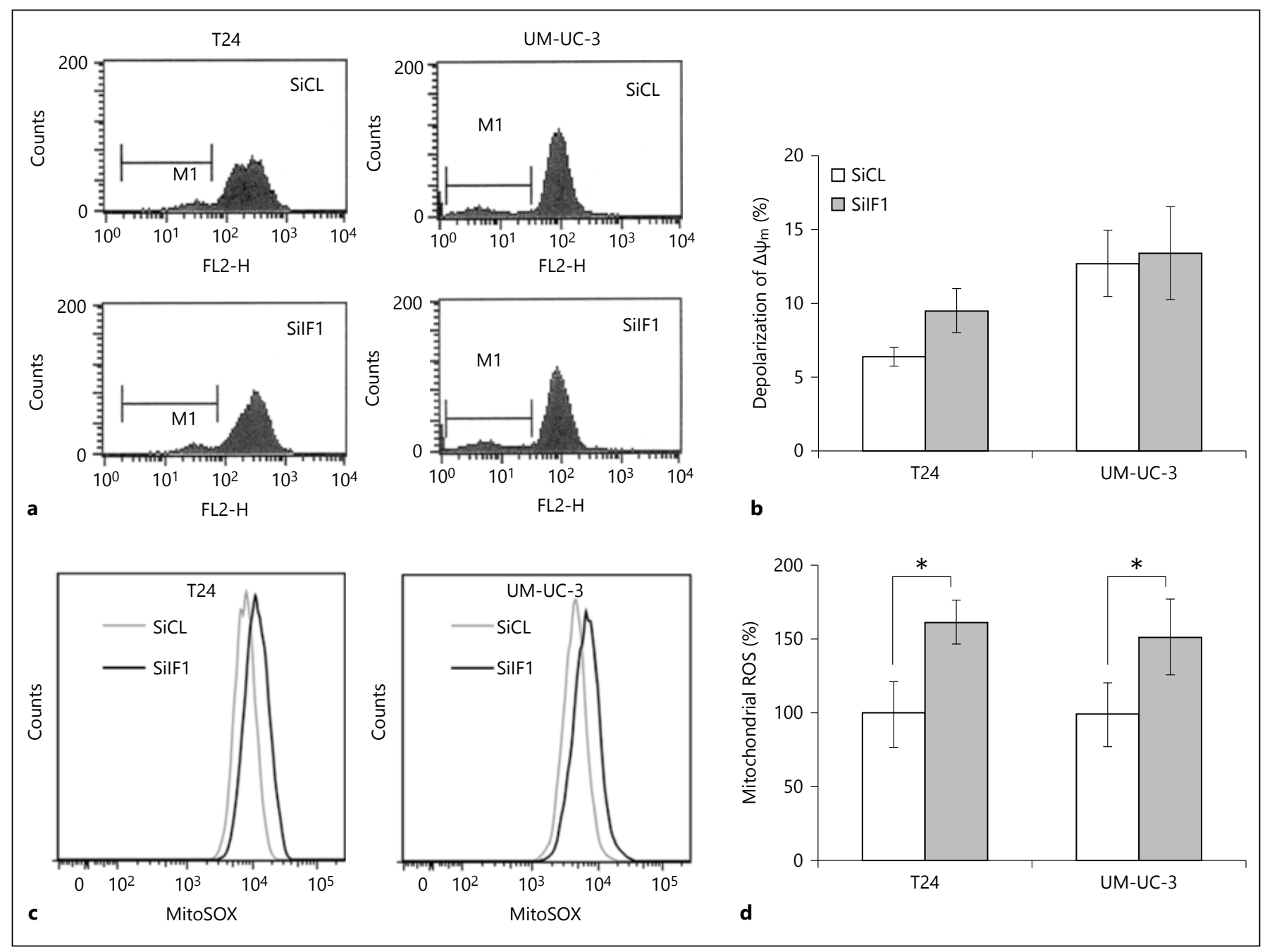

Fig. 5. Knockdown of IF1 increases BCa cell mitochondrial ROS production. a Representative dot plots in T24 and UM-UC-3 cells determined by flow cytometry after staining with TMRM. M1 = Cells of depolarization. b Knockdown of IF1 did not change $\Delta \Psi_{\mathrm{m}}$ in T24 and UM-UC-3 cells. c Representative dot plots in T24 and

types $[8-10,19,20]$. Although IF1 has been associated with tumor activity $[3,6,7]$, IF1 has not been associated with specific changes in the cell cycle previously. In the current study, we found that targeting IF1 reduced the expression of cell cycle progression regulatory factors and then induced cell cycle arrest, and finally inhibited $\mathrm{BCa}$ cell proliferation. This is the first report to show IF1 expression might play a critical role in cell cycle progression.

Aberrations in cell cycle progression occur in the majority of human malignancies [16]. The cell cycle is a highly controlled process that involves tight regulation of key molecules that allow cells to progress between phases of the cell cycle. Different combinations of cyclin and cdk
UM-UC-3 cells determined by flow cytometry after staining with MitoSOX. d Knockdown of IF1 increased mitochondrial ROS production in T24 and UM-UC-3 cells. ${ }^{*} \mathrm{p}<0.05$ vs. SiCL. The experiments were repeated three times.

subunits operate at checkpoints to integrate mitogenic and antiproliferative signals. Among them, cyclins D and E play a critical role in controlling $G_{1} / S$ transition, which are often abnormally expressed in human tumors. The present study indicates that silencing of IF1 in carcinoma cells downregulated the expression of cyclin $\mathrm{E} / \mathrm{cdk} 2$, which can lead to the phosphorylation of downstream targets for growth progression in T24 and UM-UC-3 cells, and decreased the levels of cyclin $\mathrm{D} / \mathrm{cdk} 4 / \mathrm{cdk} 6$ known as protooncogenic factors of the $G_{1} / S$ phase checkpoint in UM-UC-3 cells. But, unexpectedly, we found that cyclin D expression was increased after silencing of IF1 in T24 cells. Kang et al. [21] demonstrated that al- 
though cyclin D levels increased, the downregulation of cdk4 might result in the decrease of the cyclin $\mathrm{D} / \mathrm{cdk} 4$ complex. Likewise, the decrease in cdk4 and cdk6 might result in the downregulation of the cyclin $\mathrm{D} / \mathrm{cdk} 4 / \mathrm{cdk} 6$ complex even though cyclin D levels were increased in T2 4 cells. Therefore, $\mathrm{G}_{0} / \mathrm{G}_{1}$ arrest associated IF1 knockdown in T24 cells originates from the only downregulation of the cyclin E/cdk2 complex. As a result, the downregulation of the cyclin $\mathrm{E} / \mathrm{cdk} 2$ probably induces the downregulation of the cyclin $\mathrm{D} / \mathrm{cdk} 4 / \mathrm{cdk} 6$ complex.

The $\mathrm{p} 21$ protein is the first identified cdk inhibitor and plays a critical role in cell cycle progression [22]. It effectively inhibits cdk2, cdk4 and cdk6 by blocking kinase activity or interfering with activating phosphorylation, which have a direct function in $G_{1} / S$ transition [23]. Increases in p21 levels were associated with decreased cdk levels in UM-UC-3 cells. In contrast, the downregulation of cdk levels in T24 cells was not associated with the role of p 21 since IF1 knockdown resulted in suppressed levels. These data suggest that the control of cell cycle regulatory factors by IF1 may be cell-type specific, and other factors (p27, INK family and ERK) [23, 24], besides p21, may be involved in IF1 inhibition-mediated regulation of cell cycle regulatory factors.

In our study, we showed that IF1 knockdown delayed cell cycle progression through the $G_{1}$ phase of the cell cycle signifying cell arrest at the $\mathrm{G}_{0} / \mathrm{G}_{1}$ phase. Combined with our observation that depletion of IF1 leads to decreased cell viability and colony formation of $\mathrm{BCa}$ cells, which is not due to an increase in cell death but might be related to cell proliferation, we can conclude that knockdown of IF1 induces tumor cell growth inhibition, at least in part by causing $G_{0} / G_{1}$-phase cell cycle arrest via the downregulation of the cyclin E/cdk2 and/or cyclin D/ cdk4/cdk6 complexes (fig. 4f). As high overexpression of IF1 is found in various tumor types, the results also suggest that IF1 may be an oncogene and confer its protooncogenic properties by causing cell progression through $\mathrm{G}_{1} / \mathrm{S}$ transition via the upregulation of the cyclin E/cdk2 and/or cyclin D/cdk4/cdk6 complexes.

Overexpression of IF1 has been observed in some tumors types (e.g. breast and lung cancers) and has been associated with their prognosis, but no attention has been given to IF1 in human BCa. In our study, IF1 expression was noted to be increased in BCa tissues and cells compared with normal bladder tissues and cells. Importantly, knockdown of IF1 inhibited BCa cell growth via cell cycle arrest at the $G_{0} / G_{1}$ phase, which may be an opportunity to effectively treat $\mathrm{BCa}$ as well as delay its progression, and thus be of benefit to the patients.

ATPase Inhibitory Factor 1 in Bladder Cancer
As IF1 is predominantly located inside the mitochondrial matrix and possibly regulates mitochondrial function, we then studied the potential effect of IF1 on mitochondrial ROS. We found here that IF1 knockdown increased mitochondrial ROS, which was confirmed using MitoSOX and consistent with a previous study [4]. Increased ROS generation may originate from a reduced level of the NADH:NAD+ ratio [4], but not from the change in $\Delta \Psi_{\mathrm{m}}$ since knockdown of IF1 has no effect. Although the role of increased ROS levels by IF1 inhibition was not studied here, combined with the results of previous studies and the critical role of ROS in cell proliferation, cell cycle arrest and cell survival, it is not difficult to point out that IF1 could regulate tumor cell fate through ROS in human carcinomas. It has been demonstrated that loss of $\Delta \Psi_{\mathrm{m}}$ triggers a $\mathrm{Ca}^{2+}$-mediated cascade that leads to apoptosis. The lack of a change in $\Delta \Psi_{\mathrm{m}}$ and the absence of apoptosis induction by IF1 knockdown in our study confirm that the decrease in cell growth seen in IF1 knockdown cells is related to cell proliferation.

Based on in vitro experiments, we and other researchers showed that silencing of IF1 decreased tumor cell proliferation, while Fujikawa et al. [25] indicated that depletion of IF1 did not hamper cell growth. They suggested that the functional role of IF1 in human carcinomas may differ in cancer cells and tumor types. Although IF1 has been shown to mediate cell cycle progression in this study, it is largely unclear how IF1 activates cell cycle progression regulatory factors. We will focus on the mechanisms of IF1-mediated tumor activity and IF1-mediated cell cycle progression in detail in future studies. Considering that the clinical relevance of IF1 in human tumors is controversial and the few cases examined in our study, the clinical association between IF1 and BCa should also be further scrutinized in a larger number of samples.

Taken together, the results presented here show the importance of IF1 in human BCa as it is related to tumor cell cycle progression. We are the first to show that cell cycle arrest associated with the downregulation of IF1 was accompanied by the depletion of $\mathrm{G}_{1}$ cell cycle transition in parallel with the downregulation of key cell cycle regulators. With the ability to identify subsets of cancer patients with IF1 overexpression, antagonism of this target may provide a useful therapeutic strategy for $\mathrm{BCa}$ and other tumors.

\section{Disclosure Statement}

The authors have no conflict of interest. 


\section{References}

$>1$ García JJ, Morales-Ríos E, Cortés-Hernandez $\mathrm{P}$, Rodríguez-Zavala JS: The inhibitor protein (IF1) promotes dimerization of the mitochondrial $\mathrm{F}_{1} \mathrm{~F}_{\mathrm{o}}$-ATP synthase. Biochemistry 2006;45:12695-12703.

-2 Campanella M, Parker N, Tan CH, Hall AM, Duchen MR: $\operatorname{IF}(1)$ : setting the pace of the $F(1)$ F(o)-ATP synthase. Trends Biochem Sci 2009;34:343-350.

$\checkmark 3$ Shah DI, Takahashi-Makise N, Cooney JD, et al: Mitochondrial Atpif1 regulates haem synthesis in developing erythroblasts. Nature 2012;491:608-612.

$\checkmark 4$ Campanella M, Seraphim A, Abeti R, Casswell E, Echave P, Duchen MR: IF1, the endogenous regulator of the $\mathrm{F}(1) \mathrm{F}(\mathrm{o})$-ATPsynthase, defines mitochondrial volume fraction in HeLa cells by regulating autophagy. Biochim Biophys Acta 2009;1787:393-401.

5 Lefebvre V, Du Q, Baird S, et al: Genomewide RNAi screen identifies ATPase inhibitory factor 1 (ATPIF1) as essential for PARK2 recruitment and mitophagy. Autophagy 2013;9:1770-1779.

6 Formentini L, Sánchez-Aragó M, SánchezCenizo L, Cuezva JM: The mitochondrial ATPase inhibitory factor 1 triggers a ROSmediated retrograde prosurvival and proliferative response. Mol Cell 2012;45:731-742.

7 Song R, Song H, Liang Y, et al: Reciprocal activation between ATPase inhibitory factor 1 and NF- $\kappa \mathrm{B}$ drives hepatocellular carcinoma angiogenesis and metastasis. Hepatology 2014;60:1659-1673.

$>8$ Sánchez-Aragó M, García-Bermúdez J, Martínez-Reyes I, Santacatterina F, Cuezva JM: Degradation of IF1 controls energy metabolism during osteogenic differentiation of stem cells. EMBO Rep 2013;14:638-644.
-9 Sánchez-Cenizo L, Formentini L, Aldea M, et al: Up-regulation of the ATPase inhibitory factor 1 (IF1) of the mitochondrial H+-ATP synthase in human tumors mediates the metabolic shift of cancer cells to a Warburg phenotype. J Biol Chem 2010;285:25308-25313.

10 Sánchez-Aragó M, Formentini L, MartínezReyes I, et al: Expression, regulation and clinical relevance of the ATPase inhibitory factor 1 in human cancers. Oncogenesis 2013;2:e46.

11 Hanahan D, Weinberg RA: Hallmarks of cancer: the next generation. Cell 2011;144:646674.

12 Giacoia EG, Miyake M, Lawton A, Goodison S, Rosser CJ: PAI-1 leads to G1-phase cell-cycle progression through cyclin D3/cdk4/6 upregulation. Mol Cancer Res 2014;12:322-334.

13 Faccenda D, Tan CH, Seraphim A, Duchen MR, Campanella M: IF1 limits the apoptoticsignalling cascade by preventing mitochondrial remodelling. Cell Death Differ 2013;20: 686-697.

14 Wei S, Fukuhara H, Chen G, et al: Terrestro$\sin \mathrm{D}$, a steroidal saponin from Tribulus terrestris L., inhibits growth and angiogenesis of human prostate cancer in vitro and in vivo. Pathobiology 2014;81:123-132.

$\checkmark 15$ Polireddy K, Chavan H, Abdulkarim BA, Krishnamurthy P: Functional significance of the ATP-binding cassette transporter B6 in hepatocellular carcinoma. Mol Oncol 2011;5: 410-425.

16 Dai Y, Wilson G, Huang B, et al: Silencing of Jagged1 inhibits cell growth and invasion in colorectal cancer. Cell Death Dis 2014;5: e1170.

17 Sohn YS, Tamir S, Song L, et al: NAF-1 and mitoNEET are central to human breast cancer proliferation by maintaining mitochondrial homeostasis and promoting tumor growth. Proc Natl Acad Sci U S A 2013;110:1467614681.
18 Chen WW, Birsoy K, Mihaylova MM, et al: Inhibition of ATPIF1 ameliorates severe mitochondrial respiratory chain dysfunction in mammalian cells. Cell Rep 2014;7:27-34.

19 Bravo C, Minauro-Sanmiguel F, MoralesRíos E, Rodríguez-Zavala JS, García JJ: Overexpression of the inhibitor protein $\mathrm{IF}_{1}$ in AS30D hepatoma produces a higher association with mitochondrial $\mathrm{F}_{1} \mathrm{~F}_{\mathrm{o}}$ ATP synthase compared to normal rat liver: functional and cross-linking studies. J Bioenerg Biomembr 2004;36:257-264.

20 Chernyak BV, Dedov VN, Gabai VL: Mitochondrial ATP hydrolysis and ATP depletion in thymocytes and Ehrlich ascites carcinoma cells. FEBS Lett 1997;337:56-59.

21 Kang YJ, Chung HJ, Nam JW, et al: Cytotoxic and antineoplastic activity of timosaponin AIII for human colon cancer cells. J Nat Prod 2011;74:701-706.

22 Cmielová J, Rezáčová M: p21 Cip1/Waf1 protein and its function based on a subcellular localization. J Cell Biochem 2011;112:3502-3506.

$23 \mathrm{Hu}$ XT, Zuckerman KS: Role of cell cycle regulatory molecules in retinoic acid- and vitamin D3-induced differentiation of acute myeloid leukaemia cells. Cell Prolif 2014;47:200210.

24 Abbas T, Dutta A: p21 in cancer: intricate networks and multiple activities. Nat Rev Cancer 2009;9:400-414.

25 Fujikawa M, Imamura H, Nakamura J, Yoshida M: Assessing actual contribution of IF1, inhibitor of mitochondrial $\mathrm{F}_{\mathrm{o}} \mathrm{F}_{1}$, to ATP homeostasis, cell growth, mitochondrial morphology, and cell viability. J Biol Chem 2012; 287:18781-18787. 